\title{
Reflets
}

Revue ontaroise d'intervention sociale et communautaire

\section{Enjeux et pratique sociale en SAE : les intervenantes seront-elles les exclues de leur propre pratique?}

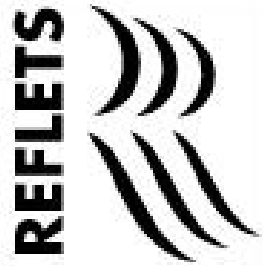

\section{Marie Drolet et Denise Ouellette}

Volume 9, numéro 2, automne 2003

Travail et mieux-être

URI : https://id.erudit.org/iderudit/011093ar

DOI : https://doi.org/10.7202/011093ar

Aller au sommaire du numéro

Éditeur(s)

Reflets : Revue ontaroise d'intervention sociale et communautaire

ISSN

1203-4576 (imprimé)

1712-8498 (numérique)

Découvrir la revue

Citer cet article

Drolet, M. \& Ouellette, D. (2003). Enjeux et pratique sociale en SAE : les intervenantes seront-elles les exclues de leur propre pratique? Reflets, 9(2), 114-138. https://doi.org/10.7202/011093ar
Résumé de l'article

Initiée en1997, la réforme ontarienne de la protection de l'enfance a modifié la pratique sociale dans les Sociétés d'aide à l'enfance (SAE). Entre autres, deux outils standardisés de prise de décision ont été introduits: le modèle d'évaluation des risques (ORAM) et le modèle de mesure du bien-être des enfants placés (Looking after children). Le concept de diffusion de l'innovation sera ici utilisé pour jeter un éclairage sur les enjeux inhérents à l'introduction de nouveautés. Ceux liés à la pratique actuelle en SAE seront aussi cernés afin de dresser un bilan critique des conditions de pratique que les intervenantes sociales y affrontent.
Tous droits réservés (C) Reflets : Revue ontaroise d'intervention sociale et communautaire, 2002
Ce document est protégé par la loi sur le droit d'auteur. L'utilisation des services d’Érudit (y compris la reproduction) est assujettie à sa politique d'utilisation que vous pouvez consulter en ligne.

https://apropos.erudit.org/fr/usagers/politique-dutilisation/ 


\title{
Enjeux et pratique sociale en SAE : les intervenantes seront-elles les exclues de leur propre pratique?
}

\author{
Marie Drolet, Ph.D, \\ École de service social, Université d'Ottawa \\ Denise Ouellette, M.S.S. \\ Société d'aide à l'enfance d'Ottawa
}

\section{Introduction}

Le marché de l'emploi a globalement changé au Canada au cours des années 1990. Nous assistons présentement à plusieurs phénomènes: la rationalisation et la restructuration des organisations, le recours à des formes de travail non conventionnelles, les changements technologiques qui modifient nos façons de faire, une polarisation entre l'exclusion du marché du travail pour les uns et l'augmentation, pour d'autres, du nombre d'heures consacrées au travail rémunéré, ce qui inclut les heures supplémentaires payées ou non. À ce chapitre, selon Higgins et Duxbury, «il semble que ce soit dans les organismes sans but lucratif (services sociaux, santé, éducation, etc.) que les exigences liées aux heures de travail supplémentaires soient les plus grandes» et que «ce sont eux qui donnent le plus de temps à l'employeur» (2002: xvi-xviii).

Cette mutation du marché du travail s'applique au milieu de la protection de l'enfance. Il s'agit d'un secteur où le service social est en transformation. Il fait face à des restructurations organisationnelles et à l'introduction massive de la technologie ${ }^{1}$. Une 
couverture médiatique négative, les transformations sociétales et les surcharges bureaucratiques soulèvent des enjeux importants pour la pratique en matière de protection des enfants ${ }^{2}$. À cet effet en 2001, les résultats d'une recherche menée par l'Association ontarienne des Sociétés d'aide à l'enfance (AOSAE) ont permis d'affirmer que les intervenantes sociales consacrent maintenant $30 \%$ de leur temps au contact direct avec les clients et $70 \%$ aux demandes bureaucratiques (Hurwitz et Cresswell 2001) ${ }^{3}$. Devant un tel constat, certains parlent d'échec de l'État à l'égard de la protection et du bien-être des enfants dans les agences de protection. À elles seules, ces raisons justifient la nécessité d'innover et d'améliorer les services offerts par les Sociétés d'aide à l'enfance (SAE).

Dans ce contexte de remise en cause, les intervenantes éprouvent des problèmes dans leurs prises de décision, en raison d'un manque de justification, d'une subjectivité et une inconsistance trop grande ${ }^{5}$ ou d'une formation insuffisante. Elles affrontent l'inefficacité de certaines de leurs interventions (Cicchinelli 1995) et les limites des ressources ${ }^{6}$. Cet enjeu majeur relance le débat entre l'art et la science qui traverse la profession du service social. Ce débat nous confronte avec le fait que la pratique de l'intervention sociale se situe aux confins de la

"L'art fait appel à la sagesse de la pratique dans les prises de décision et la science réfêre notamment à l'utilisation d'instruments empiriques..." scientificité ${ }^{7}$. L'art fait appel à la sagesse de la pratique dans les prises de décision et la science réfere notamment à l'utilisation d'instruments empiriques ${ }^{8}$. Une solution privilégiée pour solidifier les prises de décision, entre autres dans le champ de la protection des enfants en Ontario, s'avère l'introduction d'outils standardisés de collecte d'information en ce qui a trait à la clientèle. Ces outils servent par la suite de soutien à la prise de décision et à l'élaboration de plans d'intervention. Il s'agit donc d'un choix en faveur de la science que le Ministère des services sociaux et communautaires (MSSC), en collaboration avec l'AOSAE et les SAE, a favorisé en 1997, en Ontario, dans le cadre d'une réforme des services de la protection de l'enfance (Gold et al. 2001).

Cette réforme, avec toutes les modifications qui s'y rattachent, encadre les décisions des intervenantes sociales et peut donc avoir des conséquences directes sur leur pratique. Quels sont les enjeux pour les travailleuses sociales liées à l'introduction des outils 
"Ce choix, en faveur de l'empirisme, occultet-il une perte de contrôle des intervenantes sociales sur leur réalité, un glissement vers la technicité de la pratique sociale?" standardisés et des nouveaux défis qu'ils comportent pour leur pratique? À cet égard, le concept de diffusion de l'innovation permettra d'éclaircir les enjeux inhérents à l'insertion de nouveautés dans la pratique sociale. Y a-t-il d'autres aspects liés à l'organisation de la SAE qui mettent en contexte la réaction des intervenantes aux diverses nouveautés, notamment l'introduction de ces outils standardisés?

Pour répondre à ces questions, nous exposerons brièvement les changements en cours dans les SAE. Une attention spéciale sera portée à l'introduction des deux grilles standardisées de collecte de données: le modèle d'évaluation des risques d'abus (ORAM) et le modèle S'occuper des enfants (Looking after Children: $L A C$ ). Ces outils sont encadrés par la Loi sur les services à l'enfance et à la famille (LSEF), modifiée en 2000. Le concept de la diffusion de l'innovation sera par la suite circonscrit pour mieux saisir les enjeux pour les travailleuses sociales à ces innovations. En conclusion, nous posons certaines questions de fond concernant le débat de l'art ou de la science en service social, débat réintroduit par l'utilisation d'outils standardisés pour guider les prises de décision en SAE. Ce choix, en faveur de l'empirisme, occulte-til une perte de contrôle des intervenantes sociales sur leur réalité, un glissement vers la technicité de la pratique sociale? Le contexte actuel conditionne-t-il tout ce débat? Les intervenantes sociales seront-elles les exclues de leur propre pratique?

\section{Les changements apportés par la réforme}

Le MSSC a introduit, depuis 1997, certains changements qui touchent directement la pratique du service social. Ainsi, il a modifié la Loi sur les services à l'enfance et à la famille (LSEF) et intégré deux instruments standardisés pour encadrer la pratique des intervenantes en protection de l'enfance: le modèle d'évaluation des risques d'abus (ORAM) et le modèle S'occuper des enfants concernant le bien-être des enfants placés en famille d'accueil. 


\section{- La Loi sur les services à l'enfance et à la famille}

Afin de protéger les enfants victimes de maltraitance, la LSEF établit les pouvoirs d'intervention des agences de protection (The ARA Consulting Group Inc. 1998). Elle en précise les normes décisionnelles et les directives. En outre, les SAE encadrent leur pratique avec d'autres règles procédurales. La loi a été modifiée en septembre 2000, sur les points suivants: la mission, la définition opérationnelle de la maltraitance et la planification en vue d'une permanence (Hatton et al. 1998; MSSC 1999). Nous ferons référence uniquement à ces trois modifications, car elles ont un effet direct sur les grilles standardisées introduites à la SAE.

La mission : L'objet principal de la loi de 2000 devient l'intérêt véritable de l'enfant, sa protection et son bien-être. Les autres objets visés par cette loi sont renvoyés maintenant au second plan, que ce soit la préservation familiale, le plan d'action le moins perturbateur, la stabilité et le développement de l'enfant, les différences culturelles et religieuses ou le droit des Autochtones d'avoir leur propre agence de protection dans la mesure du possible (MSSC 1999). Les modifications, orientées vers les besoins et le meilleur intérêt de l'enfant, n'excluent pas pour autant les besoins des parents et le maintien de la famille.

La définition opérationnelle de la maltraitance : La définition d'un enfant ayant besoin de protection a été élargie pour mettre l'accent sur la négligence en lien avec le risque ou les maux physiques et les risques ou les maux affectifs (Trocmé et al. 1999). Cet élargissement de la définition permet maintenant une intervention plus précoce et surtout abaisse le seuil de risque à l'égard des mauvais traitements (MSSC 1999). En effet, l'expression «risque substantiel de subir des maux physiques, sexuels ou affectifs» a été remplacée par «risque vraisemblable de subir ces maux» (LSEF 2000). Il faut également noter la responsabilité qu'ont les citoyens et citoyennes à l'égard de la protection des enfants, ainsi que

"...le nombre

d'enquêtes a augmenté de $20 \%$ depuis 1997 en Ontario... » l'obligation pour tous et toutes de signaler la possibilité de mauvais traitements s'ils ont des soupçons (LSEF 2000).

À cet effet, le nombre d'enquêtes a augmenté de $20 \%$ depuis 1997 en Ontario (Hurwitz et Cresswell 2001). De plus, selon 
une recherche effectuée entre avril 2000 et mars 2001 par l'AOSAE, le nombre de cas corroborés s'est accru de $27 \%$ et le nombre d'enfants pris en charge a fait un bond de $36 \%$ depuis 1998 (OACAS 2001). Ces augmentations considérables peuvent être attribuées aux modifications à la LSEF (SAE 2000-2001).

La planification en vue de la permanence (permanency planning): La planification continue en vue de la permanence est une autre modification à la loi et elle se définit par l'établissement d'un plan de services à long terme pour l'enfant ayant besoin de protection afin de lui assurer une continuité de soins (Wilkes 2002). Ce plan doit commencer dès les premiers contacts avec une agence de protection.

Dans la foulée de la planification de l'avenir des enfants, un des changements significatifs apportés par la LSEF touche la durée des soins temporaires (MSSC 1999). Ces derniers correspondent au placement en famille d'accueil ou en foyer de groupe lorsqu'il s'agit de mieux garantir la protection des enfants en difficulté dans leur famille ou la satisfaction des besoins particuliers que le milieu familial ne peut assurer (Trocmé et al. 2000). Les soins temporaires se voient maintenant calculés sur une base cumulative et ne peuvent dépasser 12 mois pour les enfants de moins de 6 ans, et de 24 mois pour ceux âgés de 6 à 16 ans. Au-delà de cette limite, en tenant compte du meilleur intérêt de l'enfant, celui-ci doit retourner à sa famille biologique ou devenir un pupille de la $\mathrm{SAE}^{9}$ ou de la Couronne ${ }^{10}$, avec maintien de contacts avec sa famille d'origine ou possibilité d'adoption (LSEF 2000). Ainsi, afin d'assurer une permanence, une stabilité et une continuité de services, des changements sont en cours pour permettre une adoption plus rapide des enfants concernés.

La probabilité d'adoption est nettement plus élevée pour les bambins (Aitken 2002). S'il y a rupture de contacts familiaux ou impossibilité d'avoir une famille d'adoption, l'enfant est placé en famille d'accueil à long terme. Or, il existe un manque important de familles d'accueil dans certaines régions. Par conséquent, des enfants doivent vivre en foyer de groupe (Harvey 2002). Les intervenantes tentent alors d'éviter les placements multiples (le 
syndrome du "yoyo») afin d'assurer une continuité et une stabilité (Wilkes 2002 ; Aitken 2002). Ces dernières sont nécessaires pour qu'un enfant développe un attachement émotionnellement sain, de même qu'un sens d'appartenance et d'identité (Wilkes 2002; Trocmé et al. 2000).

\section{- Le modèle d'évaluation des risques}

En 1998,1'AOSAE a introduit en Ontario un modèle uniforme d'évaluation des risques (ORAM) pour la protection de l'enfance, tout d'abord mis en place à New York. Une nouvelle version a été instaurée en 2000 dans toutes les agences ontariennes de protection afin que cet outil de mesure de risques soit adapté aux modifications de la Loi sur les services à l'enfance et à la famille (LSEF). Ce modèle veut favoriser un processus décisionnel structuré et rationnel en matière de détermination de risques de mauvais traitements chez un enfant, puis de gestion de cas, sans remplacer le jugement professionnel selon le guide d'ORAM (2000). Les intervenantes utilisent donc une méthode standardisée pour les guider dans leurs prises de décision (ORAM 2000; Trocmé et al. 1999).

Le guide d'ORAM, fondé sur le modèle des facteurs de risques et des facteurs de protection, comprend trois outils: les échelles d'admissibilité,l'évaluation de la sécurité de l'enfant et l'évaluation des risques. Les échelles d'admissibilité doivent être complétées en premier afin de déterminer la poursuite ou non du processus d'investigation. L'évaluation de la sécurité de l'enfant regroupe douze questions qui fournissent un portrait précis de la situation immédiate de l'enfant et des circonstances liées à l'abus. L'évaluation des risques exige que 22 facteurs soient analysés en profondeur pour chaque parent et chaque enfant de la famille. Un portrait global de la famille supporte finalement la prise de décision eu égard à l'enfant concerné (histoire sociale, bilan des facteurs de risques perçus dans leurs interactions, facteurs de protection qui les pallient et tableau synthèse établi sur une échelle). De cette évaluation, découlera un plan d'intervention dans lequel les prospectives d'avenir, respectueuses de la culture et de l'environnement, seront dégagées. 


\section{- Le modèle S'occuper des enfants}

En vue d'une planification continue et efficiente des services, d'une qualité et d'une continuité de soins auprès des enfants placés en famille d'accueil à long terme, l'outil standardisé S'occuper des enfants a été implanté dans 26 des 52 agences de protection en Ontario (Flynn et al. 2001). Cet outil a comme objectif d'évaluer les besoins des enfants placés, de planifier leurs soins et de suivre leurs progrès (Flynn et al. 2001). La version canadienne a été introduite au milieu des années $1990^{11}$ et a été ajustée à l'Enquête longitudinale nationale sur les enfants et les adolescents (Statistique Canada 1996). L'outil évalue le développement des enfants placés en famille d'accueil ou en foyer de groupe, à partir d'un modèle de résilience qui vise à identifier des contextes où pourront évoluer les enfants selon sept dimensions: la santé, l'éducation, l'identité, les relations sociales et familiales, la présentation sociale, le développement affectif et comportemental ainsi que la capacité d'autonomie $^{12}$. Un questionnaire de 30 à 50 pages conçu pour six groupes d'âge doit être rempli une fois par année par

"... les intervenantes sociales en SAE ont vu leur réalité quotidienne se transformer au cours des dernières années. En effet, celle-ci consiste maintenant à jongler avec une augmentation $d u$ nombre d'enquêtes, du nombre d'enquêtes corroborées, un accroissement des enfants pris en charge et une surcharge de tâches bureaucratiques. " l'intervenante avec l'enfant et le parent d'accueil ${ }^{13}$. En même temps, ils doivent élaborer un plan d'intervention visant à maximiser les forces, offrir des occasions de développement ainsi que contrer les difficultés de l'enfant et dépasser son passé limitatif.

En somme, les intervenantes sociales en SAE ont vu leur réalité quotidienne se transformer au cours des dernières années. En effet, celle-ci consiste maintenant à jongler avec une augmentation du nombre d'enquêtes ${ }^{14}$, du nombre d'enquêtes corroborées ${ }^{15}$, un accroissement des enfants pris en charge ${ }^{16}$ et une surcharge de tâches bureaucratiques ${ }^{17}$. De plus, l'introduction des outils standardisés s'accompagne d'une informatisation de la pratique sociale. Est-ce que l'introduction des technologies a facilitée cette dernière ou a-t-elle augmenté les exigences du travail? À ces constats et questions s'ajoute la nature même de la pratique, c'està-dire la complexité des situations familiales, la gravité des prises de décision à l'égard des enfants, de leur sécurité actuelle et de leur bien-être et de la planification de leur avenir. Par ailleurs, les changements liés à la réforme ontarienne de la protection de l'enfance de 1997 et à la LSEF ont modifié les façons de faire en 
introduisant un net durcissement de la pratique au nom du meilleur intérêt de l'enfant. Cette forte intrusion des intervenantes dans la sphère privée doit être «justifiée et sanctionnée par la cour» (Corby 1996), d'où l'emprise d'une pratique légalisée.

\section{La définition de la diffusion de l'innovation}

Les modifications à la Loi sur les services à l'enfance et à la famille (2000) ainsi que les deux outils standardisés ORAM et LAC ont apporté des changements importants à la pratique sociale en SAE. Pour jeter un éclairage différent sur les enjeux actuels liés à ces changements implantés depuis 1997, le cadre théorique de la diffusion de l'innovation a été choisi. Ce cadre théorique vise aussi à mieux comprendre les réactions potentielles des intervenantes sociales.

Le concept de la diffusion de l'innovation a été grandement influencé par Everett Rogers (1995) pour expliquer l'intégration de la technologie dans divers domaines (Herie et Martin 2002). Shin et McClomb (1998) définissent l'innovation comme la mise en place d'un nouveau produit ou service, d'un processus imaginé et réalisé dans l'intérêt de la clientèle desservie ${ }^{18}$. L'innovation tente de résoudre des problèmes en implantant d'autres façons de faire (Pearlmutter 1998). La notion de diffusion désigne le processus de transmission de l'innovation, de l'information entre les membres du système concerné (Rogers 1995).

\section{Les types de changements organisationnels}

Pearlmutter (1998) identifie deux types de changements organisationnels: graduel et transformateurs. Les changements graduels (traduction libre de incremental change) visent à modifier une procédure ou à faire des ajustements. Les changements qui altèrent 
la structure, l'orientation et les stratégies d'organisation sont de nature transformatrice (traduction libre de transformational change). Dès lors, les changements implantés dans les agences de protection en Ontario depuis 1997 sont du type graduel, car ils ont modifié les procédures au lieu de changer la structure fondamentale des SAE ou sa fonction sociale de protection des enfants.

De plus, les changements dans les agences de protection sont généralement initiés par des pressions externes, comme une nouvelle loi, (Cohen 1999; Schaffner-Goldberg 1995; Shin et McClomb 1998). Ils peuvent aussi résulter d'événements spécifiques (par exemple, des décès d'enfants connus dans les agences de protection) qui soulignent les lacunes organisationnelles et provoquent un urgent besoin d'agir pour combler celles-ci (Cohen 1999). Ils surviennent souvent en réaction à l'information négative véhiculée par les médias (Carter 2000).

\section{Qui sont les innovateurs?}

"...c'est souvent le

MSSC, subventionnant les services de protection des enfants, qui décide des changements à implanter dans les agences ontariennes. "
Certains auteurs, tels Cohen (1999), Cohen et Austin (1994), Rice (1973) et Schaffner-Goldberg (1995) constatent que les personnes centrales dans l'implantation des changements œuvrent aux échelons supérieurs de la hiérarchie. Par ailleurs, Pearlmutter (1998) souligne que tous les individus (employées, superviseures, administrateurs et membres exécutifs) ont une responsabilité face à l'introduction de changements. Ainsi, les employées engagées dans les démarches ressentent plus de motivation et une perception de contrôle sur leur réalité ${ }^{19}$, voire d'un empowerment. La perception de contrôle vise l'autonomie au travail et la participation aux prises de décision (Jex 1998).

Cela dit, dans la majorité des agences de protection, ce sont les échelons supérieurs de l'organisation qui ont un pouvoir sur les employées (Pine et al. 1998). Plus précisément, c'est souvent le MSSC, subventionnant les services de protection des enfants, qui décide des changements à implanter dans les agences ontariennes, elles dont la survie économique en dépend (Carter 2000). Ce type 
de structure de pouvoir se traduit par un sentiment d'impuissance, de vulnérabilité et de perte de contrôle chez les intervenantes sociales (Shera dans Pine et al. 1998). Or, ce sentiment est une cause importante de stress (Jex 1998). Dans de tels contextes autoritaires, les changements se font plus difficilement car les intervenantes sociales se sentent frustrées et résistent (Cohen et Austin 1994). Pine et al. (1998) stipulent que les gestionnaires doivent davantage encourager la participation des intervenantes dans l'infrastructure organisationnelle afin de maximiser l'innovation.

\section{Les prises de décision au cours du processus de l'innovation}

"...le danger

d'introduire et

d'implanter des

changements soudains, sans période d'essai, peut entraîner des conséquences inattendues, de la résistance et créer du chaos dans

l'organisation."
Une période d'essai dans le processus d'adoption ou de rejet d'une nouvelle idée peut être avantageuse dans la planification stratégique de l'innovation ${ }^{20}$. Rogers (1995) soulève qu'effectivement, une telle période peut amener un individu ou une équipe à adopter l'innovation plus rapidement. Cohen (1999) constate que le danger d'introduire et d'implanter des changements soudains, sans période d'essai, peut entraîner des conséquences inattendues, de la résistance et créer du chaos dans l'organisation. En contrepartie, un soutien accru aux nouvelles employées et davantage de formation continue peuvent être des moyens pour surmonter ces obstacles.

Par ailleurs, Rogers (1995) présente trois types de décisions liées à l'introduction de l'innovation dans une organisation. Le premier type correspond à une stratégie de donner le choix à chaque membre. Le second repose sur une stratégie consensuelle qui nourrit chez les employées un sentiment d'inclusion et de collaboration (Carter 2000). Enfin, le dernier, de type autoritaire, fait référence aux individus qui détiennent un certain pouvoir, un statut ou une expertise technique et imposent le nouveau procédé. Cette dernière figure est le cas de la plupart des changements liés au contexte légal des agences de protection. 


\section{Le succès de l'innovation}

"...de façon

quotidienne, ce sont les intervenantes sociales dans les agences de protection qui sont aux prises avec la complexité, l'imprévisibilité et la réalité de la problématique des mauvais traitements à l'égard des enfants et celle de leur placement."
Ainsi, le taux de succès de l'innovation dans des organisations de services humanitaires semble être relié aux compétences des gestionnaires et à un style de leadership participatif caractérisé par l'inventivité, le respect et la capacité à prendre des risques (Shin et McClomb 1998). Pour sa part, DePanfilis (cité dans Carter 2000) affirme que le succès sera maximisé si les superviseures des intervenantes sociales sont engagées et actives dans le processus de l'innovation. De plus, le partage des prises de décision (Cohen et Austin 1994) et la collaboration (Pine et al. 1998) entre les échelons supérieurs et les employées (la décentralisation) encouragent l'innovation.

Selon les diverses variables, par exemple, les caractéristiques organisationnelles, les conditions environnementales et les styles de leadership, explorées par Shin et McClomb (1998), seules les prises de décision décentralisées sont associées à une acceptation de l'innovation. Rogers (1995) définit la décentralisation comme étant le partage du pouvoir et du contrôle par les employées. Or, de façon quotidienne, ce sont les intervenantes sociales dans les agences de protection qui sont aux prises avec la complexité, l'imprévisibilité et la réalité de la problématique des mauvais traitements à l'égard des enfants et celle de leur placement (Pine et al. 1998).

\section{L'incertitude de l'innovation, les barrières invisibles et son insuccès}

Puisque les changements apparaissent comme complexes, voire chaotiques, il est important de conserver une stabilité, de rattacher le nouveau à quelque chose de connu ou de permettre une assez longue période d'essai (Gummer 1995; Pearlmutter 1998), 
d'anticiper les réactions et de partager de l'information (Rogers 1995) avec tous les membres. Comme le soulève Carter (2000), aucun changement ne devrait être entrepris sans avoir analysé son impact sur l'organisation entière afin de minimiser le bouleversement. À cet effet, Cohen (1999) note trois difficultés principales. D'abord, l'idée que le changement peut seulement être effectué par les échelons supérieurs. Ensuite, les gestionnaires n'ont pas les moyens d'offrir des récompenses aux membres du personnel qui soumettent de bonnes idées afin de mieux répondre aux lacunes organisationnelles. Enfin, les gestionnaires craignent qu'encourager leurs subordonnées à participer au processus d'innovation soit perçu comme un élément menaçant et perturbateur (Cohen 1999).

\section{La résistance envers l'innovation}

"...la réticence et

l'hésitation des intervenantes sociales dans les processus d'innovation sont interprétées par les gestionnaires comme étant de la

résistance. "
Dans les organisations humanitaires, on retrouve de façon inhérente une dynamique pour maintenir le statu quo et il peut se transformer en résistance au changement (DePanfilis 1996). La résistance se définit «comme des actes intentionnels ou des omissions qui défient les désirs des autres» (traduction libre, Ashforth et Mael 1998: 90). Or, les conduites de résistance ont plus de chances d'apparaître lors de situations imposées (Ashforth et Mael 1998).

Markus (1990) constate que la réticence et l'hésitation des intervenantes sociales dans les processus d'innovation sont interprétées par les gestionnaires comme étant de la résistance. Comme le souligne Davidson (1990), les intervenantes sociales doivent accepter que l'amélioration des pratiques sociales passe nécessairement par la reconnaissance de leurs blocages et des lacunes de la profession. Selon cet auteur, le doute ou le questionnement sur les interventions est un élément indispensable du processus d'acceptation de nouvelles techniques, l'apprentissage à partir des questionnements et des erreurs étant le fondement même de la méthode scientifique. Cette résistance au changement peut se 
manifester de plusieurs façons: les intervenantes auront tendance à changer d'emploi, à s'absenter, à baisser leur productivité et leur engagement. Il y aura de l'animosité, un syndicalisme plus grand et plus actif (Carter 2000).

Davidson (1990) discute de quatre moyens pour surmonter la résistance au changement dans une organisation. Premièrement, le raisonnement derrière l'innovation doit être présenté aux employées. Deuxièmement, l'adoption de l'innovation est favorisée par une organisation qui accorde de l'importance à une communication ouverte, ceci par l'utilisation de moyens comme la supervision, la formation et les groupes de soutien pour les intervenantes sociales. Troisièmement, la flexibilité envers les employées stimule leur coopération et leur engagement. Une étude ontarienne explorant les dimensions nécessaires pour l'obtention de l'excellence a démontré que la flexibilité encourageait la créativité et la résolution des problèmes (Harvey 1998). Finalement, la possibilité qu'ont les membres de l'organisation d'exprimer leurs réactions face à un changement et l'éducation de ceux-ci sur la nécessité des changements peuvent être des formes de soutien dans le processus de l'innovation.

De plus, des objectifs réalistes, atteignables et concrets facilitent l'identification des barrières dans le processus d'introduction des changements et maximisent le succès. L'appui des employées peut être obtenu si la raison des changements est clairement expliquée. Elles seront plus motivées à accepter les changements proposés si les impasses du maintien du statu quo leur sont communiquées (Carter 2000). Selon Bargal et Schmid (cité dans Carter 2000), dans ce processus, les intervenantes sociales doivent être assurées que leurs connaissances et leurs valeurs sont respectées et que les changements proposés sont dans le meilleur intérêt des enfants et en faveur d'une planification en vue de leur permanence. Par ailleurs, Davidson (1990) constate que les intervenantes qui reconnaissent leurs incertitudes envers l'innovation peuvent plus facilement évoluer d'une position de doute à une ouverture aux nouvelles idées.

En définitive, l'innovation est un processus qui demande beaucoup de temps et d'énergie (Rogers 1995), de la patience et 
de la persévérance de la part de tous (Cohen et Austin 1994). Comme nous l'avons constaté, les changements graduels sont adoptés plus facilement que ceux visant une transformation structurelle; ils le sont quand le pouvoir est partagé (à un certain degré) entre les membres de l'organisation. La complexité des problématiques et des processus d'innovation dans les agences de protection exige qu'une attention toute particulière soit apportée afin de mieux comprendre et respecter la réalité quotidienne des intervenantes sociales. Il importe de mettre en lumière certains enjeux qu'elles affrontent à tous les jours et qui pourraient interférer dans leur ouverture à des changements, donc nourrir une résistance elle-même inhérente à tout changement.

\section{Enjeux de la pratique sociale en SAE : un défi supplémentaire à la diffusion d'innovation}

Afin de mettre en contexte et en vue d'une compréhension plus globale de la réalité et des réactions des intervenantes sociales à la diffusion d'innovation dans les SAE, nous examinerons les enjeux inhérents aux conditions d'imputabilité et les débats entourant les deux outils standardisés, ORAM et S'occuper des enfants. Puis, nous ferons un bref état de leur situation de stress.

\section{- Les conditions d'imputabilité}

La pression du gouvernement en faveur d'une plus grande imputabilité a clairement joué un rôle important dans la réforme du bien-être de l'enfance en Ontario depuis 1997 (Hurwitz et Cresswell 2001). L'imputabilité est définie comme la responsabilité légale et les erreurs de jugement professionnel des employées d'une agence de protection (Wimpfheimer 1993). La responsabilité professionnelle est plutôt liée à l'obligation éthique et morale d'intervenir pour le bien-être et l'intérêt véritable de l'enfant, sa sécurité et son avenir (Gove 1995). En outre, la problématique des médias qui alarment l'opinion publique sur les limites des 
agences sociales à assurer le bien-être et la protection des enfants victimes de maltraitance, met de la pression au quotidien sur les intervenantes sociales. Cette pression, relayée par les politiciens, s'exerce pour augmenter leur imputabilité et leur responsabilité professionnelle (Cicchinelli 1995). Comme le notent Hurwitz et Cresswell (2001), les tâches effectuées par les intervenantes sociales sont déjà assez complexes en raison des problématiques de maltraitance et de placement des enfants; elles se compliquent encore davantage avec une augmentation de cette imputabilité et des critiques des médias. Dans ce contexte, les changements réalisés avec la réforme ont augmenté leur charge de travail (Hurwitz et Cresswell 2001).

De surcroit, les prises de décision et les interventions des intervenantes sociales sont assujetties à un examen minutieux (par exemple, la vérification des dossiers par le MSSC) en raison des exigences d'imputabilité dans les agences de protection (Regehr et al. 2002). Ce contexte, qui pourrait vouloir dire une possibilité de poursuite judiciaire, avive l'anxiété des intervenantes qui craignent également de devenir des boucs émissaires pour les lacunes du système (Davies et al. 1999). La peur d'être prise en défaut et la pression liée à la responsabilité légale se décèlent chez les intervenantes dans leurs prises de décision dans le cadre de la gestion de cas (Regehr et al. 2002). Or, les prises de décision requises pour assurer la protection d'un enfant sont en soi difficiles et complexes (Baird et al. 1999; Dawson 2001). De plus, leur faible fiabilité est un problème (Gold et al. 2001) et elles peuvent être inconsistantes (DePanfilis et Zuravin 1999).

\section{- Le débat entourant les outils standardisés d'évaluation des risques}

Les outils standardisés d'évaluation des risques ont justement été implantés dans les agences de protection afin de favoriser cette uniformité dans les prises de décision (Baird et al. 1999; Macmillan et al. 2002). Ces outils peuvent devenir une réponse aux exigences d'imputabilité. À cet effet, l'implantation des modèles d'évaluation des risques dans les agences a pris de l'ampleur dans la dernière décennie, notamment au Canada et aux États-Unis ${ }^{21}$. Ces outils 
sont là pour vérifier si l'enfant a droit aux services, pour déterminer le seuil de gravité de l'abus et pour aider à rendre les prises de décision plus consistantes (DePanfilis et Zuravin 1999).

Dans quelle mesure l'utilisation d'outils standardisés peut-elle améliorer les prises de décision des intervenantes? Ces outils sont uniformes, construits à partir de critères spécifiques et fondés sur des écrits scientifiques sur le sujet (Cicchinelli 1995; Dawson 2001). Pour Macmillan et al. (2002), l'outil établit une liste standardisée de facteurs dans l'évaluation des risques de mauvais traitements. Il s'avère un moyen concret et rationnel pour les intervenantes afin qu'elles établissent la priorité des éléments clés dans la démarche de la gestion de cas. Il sert d'outil de formation et de supervision pour les intervenantes en début de carrière ${ }^{22}$. Il assure la responsabilité professionnelle (Macmillan et al. 2002). Il facilite la planification des plans d'intervention et le processus de fermeture des dossiers (DePanfilis 1996; Rittner 2002).

Les outils standardisés reçoivent, par contre, des critiques sévères. Le recours à de tels modèles transforme la pratique sociale en une forme plus officielle et sophistiquée à l'égard des enquêtes et de la gestion de cas de protection (Macmillan et al. 2002). Ce ne sont pas des instruments parfaitement exacts; il ne peuvent empêcher certains enfants identifiés comme n'ayant pas besoin de protection d'être maltraités ${ }^{23}$. Des auteurs prétendent que les outils discriminent, isolent et oppriment les familles vivant sous le seuil de la pauvreté. La fiabilité et la validité des outils psychométriques ne seraient pas assez bien mesurées ${ }^{24}$. Les valeurs personnelles et professionnelles des intervenantes peuvent influencer et avoir un effet sur la collecte et l'analyse des données. De plus, ces outils augmentent la tâche de travail des intervenantes étant donné les modifications apportées à la LSEF et tous les changements introduits dans les agences de protection depuis la réforme de 1997 (Macmillan et al. 2002).

Macmillan et al. (2002) concluent que l'outil d'évaluation des risques est aussi efficace que le sont les intervenantes qui le complètent. Ces auteurs mettent en lumière le danger que le modèle d'évaluation des risques soit perçu comme étant un moyen à moindres coûts pour remplacer l'éducation, la supervision et la 
formation des intervenantes au lieu d'un atout pour la formation et la supervision de ces dernières, au lieu d'un soutien au jugement clinique et à leurs compétences d'évaluation de situation ${ }^{25}$. Il est primordial que ces outils soient le complément du jugement clinique au lieu de s'y substituer ${ }^{26}$. En somme, la complexité dans le processus de prise de décision ne peut être remplacée par des approches mécanistes, technicistes (Corby, 1996), des listes récapitulatives ou d'autres solutions simples (Rittner 2002).

\section{- Le débat concernant l'outil standardisé S'occuper des enfants}

En ce qui concerne la planification en vue de la permanence, l'implantation du modèle S'occuper des enfants signifie qu'il y a des lacunes au sein du système de protection, lequel doit offrir une stabilité aux enfants pris en charge ${ }^{27}$ et placés en famille d'accueil. Or, la documentation fait état de désavantages liés à ce modèle. Le format des questionnaires où il faut cocher la réponse peut être perçu comme intimidant et créer une distance entre les intervenantes et l'enfant ${ }^{28}$. Ces questionnaires sont plutôt impersonnels et ne facilitent pas toujours la communication ${ }^{29}$. Selon Garrett (1999), ils sont trop directs, semblables à un interrogatoire et pouvant étiqueter les enfants en renforçant la stigmatisation à l'égard de leurs difficultés (Knight et Caveney 1998). Pourtant, S'occuper des enfants est fondé sur un modèle de résilience axé sur les occasions de développement, de même que sur un plan d'intervention dynamique centré sur les forces de l'enfant concerné. Pour d'autres, cet outil revêt un caractère trop académique et technocratique facilitant le suivi de la clientèle, l'analyse et la vérification des dossiers (Garrett 1999).

\section{- Le stress vécu par les intervenantes sociales}

En 2000, une recherche ontarienne menée par Regehr et al. (2000) a étudié les diverses sources de stress des intervenantes sociales dans le domaine de la protection de l'enfance. Les résultats mettent en perspective certaines sources de stress qui découlent directement des changements apportés au cours de la réforme ontarienne. Selon Regehr et al. (2000), 75 \% des participantes à la 
recherche ont relié le stress à la [sur]charge de travail, $60 \%$ à la documentation et aux délais qui leur sont impartis, 50,6 \% aux changements organisationnels et 33,7\% à l'imputabilité. À cet effet, la prééminence de l'aspect légal semble se répandre dans le discours des intervenantes sociales. Elles sont constamment averties que leurs prises de décision dans la gestion de cas pourraient avoir un effet sur leur crédibilité, même résulter en une poursuite judiciaire. Or, l'analyse des situations problèmes est faite en scrutant un seul dossier, et non pas en fonction de la charge globale de travail qu'affronte une intervenante, ce qui accroît les exigences d'imputabilité.

De plus, Regehr et al. (2000) avancent que le stress augmente les difficultés personnelles et les problèmes de santé des intervenantes. La double fonction d'aide et d'enquête peut créer des conflits de rôles et des ambiguïtés, raison d'un haut degré d'épuisement professionnel ${ }^{30}$. Rappelons que le sentiment de perte de contrôle sur son travail est une cause importante de stress (Jex 1998). Or, les intervenantes rapportent un degré de stress et d'épuisement professionnel très élevé (Davies et al. 1999). On doit donc s'interroger quant aux conditions de travail dans les agences de protection, quant aux conséquences des outils standardisés sur la pratique sociale et sur le quotidien des intervenantes.

La réalité quotidienne des intervenantes en SAE, qui consiste à jongler avec l'augmentation du nombre d'enquêtes, l'accroissement des enfants pris en charge et la surcharge de tâches bureaucratiques est inquiétante. Les intervenantes vivent alors du stress relié à la [sur]charge de travail, à la documentation précise des prises de décision et aux délais qui leurs sont impartis, à l'imputabilité accrue et aux changements organisationnels. À ces constats s'ajoutent la complexité des situations familiales, des mesures de risques pour les enfants, de la planification en vue de la permanence ainsi que les pressions de l'opinion publique et des médias. À l'instar des profondes transformations actuelles du marché du travail, les agences de protection doivent, de plus, innover et s'améliorer dans le sens d'une rationalisation efficiente et de l'introduction massive des technologies. Une solution 
privilégiée s'avère nettement l'implantation des outils standardisés d'évaluation des risques (ORAM) et de mesure de bien-être des enfants placés (S'occuper des enfants), outils qui soutiennent les prises de décision et l'élaboration de plans d'intervention. Il s'agit là d'un choix clair en faveur de la science dans le débat entre l'art et la science de la pratique en service social.

Outre le contexte de la diffusion des innovations, les bureaucraties créent une hiérarchie à l'intérieur de laquelle les intervenantes ont peu de contrôle sur les prises de décision organisationnelles. Par contre, une participation des intervenantes aux prises de décision, voire leur empowerment, faciliterait l'adoption et même le succès de l'implantation d'innovations; dans le cas contraire, il peut apparaître une résistance de leur part. Pour contrer cette résistance, on doit persuader les travailleuses sociales que les changements proposés le sont dans l'intérêt véritable des enfants et en faveur d'une meilleure planification continue de services, que leurs connaissances, leurs valeurs, leur éthique et leur essence seront respectées.

Or, introduire de nouveaux outils standardisés ne vient-il pas questionner la nature même du service social? Les questions soulevées par l'emploi, par la SAE, d'outils spécialisés mettent sur la table les débats entre l'art et la science en service social, de même que le débat plus récent à l'égard de la pratique basée sur l'évidence (traduction libre de evidence-based practice) (Sheldon 2001; Webb 2001). D'une part, certains défendent que la plupart des décisions en service social sont uniquement basées sur des opinions, donc biaisées, et que l'instrumentation empirique construite sur des recherches quantitatives et aléatoires (Sheldon 2001) et des pratiques évaluées comme efficientes (Reid 2002) doivent supplanter ces limites. D'autres avancent au contraire que, pour être complètes, les décisions doivent inclure l'art, soit cette sagesse de la pratique, l'unicité des clients perçus comme acteurs sociaux, le contexte et la complexité de leurs situations ${ }^{31}$.

Ce choix en faveur de l'empirisme peut entraîner un glissement de la pratique sociale vers des opérations routinières (Webb 2001), vers une technicité à l'instar de l'informatisation massive (Freitag 2002), surtout dans un contexte de surcharge de travail, la 
rationalisation économique traverse alors tout ce débat (Webb 2001). À cet effet, comment ces outils standardisés transformentils vraiment le quotidien des intervenantes et leur autonomie, leur pratique quotidienne auprès des clients? Une résistance de la part des intervenantes sociales gronde-t-elle? Elles doivent se donner des lieux de parole, de regards critiques et de stratégies d'action, voire d'emprise et d'empowerment. Sinon, les travailleuses sociales vont devenir les exclues de leur propre pratique.

\section{Bibliographie}

AITKEN, G. (2002). «Extending options in permenancy planning», dans Sparrow Lake Alliance, Permanency Planning in the Child Welfare, Children in Limbo Task Force.

ARONSON, J. et S. SAMMON (2000). «Practice amid social service cuts and restructuring:Working with the contradictions of "small victories "”, Canadian Social Work Review, vol. 17, no 2, 167187.

ASHFORTH, B. E. et F. A. MAEL (1998). «The power of resistance: Sustaining valued identities», dans R. M. Kramer et M. A. Neale, éd., Power and Influence in Organizations, California, SAGE Publications, 89-119.

BAIRD, C., D.WAGNER, T. HEALY et K.JOHNSON (1999). «Risk assessment in child protective services: Consensus and actuarial model reliability", Child Welfare, vol. LXXVIII, no 6, 723-748.

BEAUMONT, B (1999). «Risk assessment and prediction research», dans P. Parsloe ,éd., Risk assessment in social care and social work, London, Jessica Kingsley Publishers.

BURKE, P (1999). "Social services staff: Risks they face and their dangerousness to others», dans P. Parsloe , éd., Risk assessment in social care and social work, London, Jessica Kingsley Publishers.

CALLAHAN, M. (2001). «Débat. L'évaluation du risque dans les services de protection de l'enfance. Non: Ces outils [...] ne réduisent pas le risque pour les enfants», Revue canadienne de service social, vol. 18, no 1,171-177.

CAMILLERI, P. (1999). «Social work and its search for meaning:Theories, narratives and practices», dans B. Pease et J. Fook , éd., Transforming Social Work Practice. Postmodern Clinical Perspectives, New York et Londres, Routledge, 25-39.

CARTER, J. (2000). Implementing change in child protection agencies: The case of the Ontario risk assessment model at Children's aid society "X», thèse de maitrise en service social, Montréal, Université McGill.

CASH, S. J. (2001). «Risk assessment in child welfare:The art and science», Children and Youth Services Review, vol. 23, no 11,811-830.

CICCHINELLI,L. F. (1995). «Risk assessment: Expectations and realities», The APSACAdvisor, vol. 8, no 4, 3-8.

Cloutier, R., G. CARRIER, K. Viel, G. PAQUet, D. LESSARD, A. NAHID, A.-M. BARRETTE et M. LASNIER (2001). Projet d'intégration clinique. Intégration d'outils informatisés à l'intervention, Beauport, Centre Jeunesse de Québec, Institut universitaire. 
COHEN, B. J. (1999). «Fostering innovation in a large human services bureaucracy», Administration in Social Work, vol. 23, no 2, 47-59.

COHEN, B. J. et M. J. AUSTIN (1994). «Organizational learning and change in a public child welfare agency», Administration in Social Work, vol. 18, no 1,1-19.

CORBY, B. (1996). «Risk assessment in child protection work», dans H. Kemshall et J. Pritchard, éd., Good practice in risk assessment and risk management, London, Jessica Kingsley Publishers.

DAVIDSON, K.W. (1990). «Doubt as a source of innovation in developing effective services to families", Families in Society. The Journal of Contemporary Human Services, vol. 71, no 5, 296-302.

DAVIES, L., M. McKINNON, P. RAINS et L. MASTRONARDI (1999). «Rethinking child protection practice:Through the lens of a voluntary service agency», Canadian Social Work Review, vol. 16 , no $1,103-116$.

DAWSON, R. (2001). «Débat: L'évaluation du risque dans les services de protection de l'enfance, Oui:l'évaluation du risque fait partie intégrante de la pratique», Revue canadienne de service social, vol. 18, no 1, 165-169.

DePANFILIS, D. et S. J. ZURAVIN (1999). «Epidemiology of child maltreatment recurrences», Social Service Review, vol. 73, no 2, 218-239.

DePANFILIS, D. (1996). «Implementing child mistreatment risk assessment systems: Lessons from theory", Administration in Social Work, vol. 20, no 2, 41-59.

FLYNN, R., H. GHAZAL, S. MOSHENKO et L.WESTLAKE (2001). «Main features and advantages of a new, 'Canadianized' version of the assessment and action record from Looking After Children", OACAS Journal, vol. 45, no 2,1-5.

FREITAG, M. etY. Bonny (coll.) (2002). L'oubli de la société. Pour une théorie critique de la postmodernité, France, Les Presses de l'Université Laval.

GAMBRILL, E. et A. SHLONSKY (2001). «The need for comprehensive risk management systems in child welfare», Children and Youth Services Review, vol. 23, no 1,79-107.

GARRETT,P.M. (1999). «Mapping child-care social work in the final years of the twentieth century: A critical response to the 'Looking after children' system», British Journal of Social Work, vol. 29, 27-47.

GOLD, N., R. BENBENISHTY et R. OSMO (2001). "A comparative study of risk assessments and recommended interventions in Canada and Israel», Child Abuse \& Neglect, vol. 25, 607-622.

GOLD, N (1999). "The nature and function of assessment», dans F. TURNER (dir.), Social work practice: A Canadian perspective, Scarborough, Prentice Hall Allyn and Bacon Canada.

GOVE, T. J. (1995). «Children are people too: The challenge for change in child welfare», Journal of the Ontario Association of Children's Aid Societies, vol. 31, no 3,18-21.

GUMMER, B. (1995). «Reinventing, restructuring, and the big bang theory of organizational change», Administration in Social Work, vol. 19, no 3, 83-97.

HARVEY, B. (2002). «Foster home numbers dwindle as need rises», Ottawa Citizen, 16 déc., A1-A2.

HARVEY, C. (1998). «Defining excellence in human service organizations», Administration in Social Work, vol. 22, no 1,33-45.

HATTON, M.-J.et al. (1998). Protecting vulnerable children: Report of the panel of experts on child protection. Toronto, Ministère des services sociaux et communautaires.

HERIE, M. et G. W. MARTIN (2002). «Knowledge diffusion in social work: A new approach to bridging the gap», Social Work, vol. 47, no 1, 85-95. 
HIGGINS, C. et L. DUXBURY (2002). Enquête nationale sur le conflit entre le travail et la vie personnelle (2001): Rapport 1, Santé Canada.

HURWITZ, H., et D. CRESSWELL (2001). "Workload measurement project report», OACAS Journal, vol. 45, no 1,8-38.

JACKSON, S (1998). «Looking after children: A new approach or just an exercise in formfilling? A response to Knight and Caveney», British Journal of Social Work, vol. 28, 45-56.

JEX, S. M. (1998). Stress and Job Performance. Theory, Research and Implications for Managerial Practice, California, SAGE Publications.

JOHNSON, M. A., C. HENDRICKS BROWN et S. J. WELLS (2002). «Using classification and regression trees (CART) to support worker decision making», Social Work Research, vol. 26, no 1, mars, 19-29.

KNIGHT, T et S. CAVENEY (1998). "Assessment and action records: Will they promote good parenting?», British Journal of Social Work, vol. 28, 29-43.

LBEC - Ligue pour le bien-être de l'enfance du Canada (2002). «S'occuper des enfants: mise à jour canadienne", Journal de LBEC, vol. 1, no 1,1-6.

Loi sur les services à l'enfance et à la famille (LSEF), 1990 [2000]. chap. C.11.

LYONS, P., H. J. DOUECK et J. S. WODARSKI (1996). «Risk assessment for child protective services: A review of the empirical literature on instrument performance», Social Work Research, vol. 20, no 3, 143-155.

MacMILLAN, H., P. D. STEINHAUER et D. CHAPPEL (2002). "Use of risk assessment tools», dans Permanency planning in the child welfare system, Sparrow Lake Alliance.

MARKUS, E. J. (1990). «Computerization: A precondition for, or a product of, responsible social work practice», International Journal of Sociological and Social Policy, vol. 10, no 4-6, 30-53.

MSSC, Ministry of Community and Social Services (1999). Handout: CFCA Amendments, Government of Ontario.

MARTINEZ-BRAWLEY, E. E. et P. M.-B. ZORITA (1998). «At the edge of the frame: Beyond science and art in social work», British Journal of Social Work, vol. 28, 197-212.

NORGAARRD,V. et S. BALLA (2002). «Looking after children Canadian-style», OACAS Journal, vol. 46, no 1,3-4.

OACAS (2001). (Page consultée le 1er décembre 2003). «Ontario child welfare statistics», [En ligne]. Adresse URL: http://www.oacas.org/resources/casstats.htm

ORAM (2000). Risk assessment model for child protection in Ontario, Ontario Association of Children's Aid Societies.

OSMO, R. et A. ROSEN (2002). «Social workers' strategies for treatment hypothesis testing», Social Work Research, vol. 26, no 1, mars, 9-18.

PAQUet, G., G. CARRIER, R. ClOUtier, F. GAGNON, D. LACERTE, A. NAHID et G. ALAIN (2001). Consultation sur l'implantation du Projet Intégration Jeunesse (PIJ) dans les quatre premiers centres jeunesse en déploiement, Beauport, Centre Jeunesse de Québec, Institut universitaire.

PEARLMUTTER, S. (1998). «Self-efficacy and organizational change leadership», Administration in Social Work, vol. 22, no 3, 23-38.

PINE, B. A.; R.WARSH et A. N. MALUCCIO (1998). «Participatory management in a public child welfare agency: A key to effective change», Administration in Social Work, vol. 22, no 1,19-32. 
PROCTOR, K. (2002). «Decision making in social work practice», Social Work Research, vol. 26, no 1, mars, 3-6.

REGEHR, C., M. M. BERNSTEIN, et K. KANANI (2002). «Liability for child welfare social workers: Weighing the risks», OACAS Journal, vol. 46, no 1, 32-39.

REGEHR, C., B. LESLIE, P. HOWE et S. CHAU (2000). (Page consultée le $1^{\text {er }}$ décembre 2003). «Stressors in child welfare practice», Bell Canada Child Welfare Research Unit, [En ligne].Adresse URL: www.cecw-cepb.ca/Pubs/PubsOther.html

REID, W. J. (2002). «Knowledge for direct social work practice: An analysis of trends», Social Service Review, vol. 76, no 1, mars, 6-33.

RICE, R. M. (1973). «Organizing to innovate in social work», Social Casework, vol. 54, no 1, 20-26.

RITTNER, B. (2002). "The use of risk assessment instruments in child protective services case planning and closures», Children and Youth Services Review, vol. 24, no 3, 189-207.

ROGERS, E. M. (1995). Diffusion of innovations, New York, The Free Press.

SAE (2000-2001). «Rapport annuel 2000-2001», Ottawa, La société de l'aide à l'enfance d'Ottawa.

SARGENT, K (1999). "Assessing risks for children», dans P. Parsloe ,éd., Risk assessment in social care and social work, London, Jessica Kingsley Publishers.

SCHAFFNER-GOLDBERG, G. (1995). «Theory and practice in program development:A study of the planning and implementation of fourteen social programs», Social Service Review, vol. 69, no 4, 614-655.

SHELDON, B. (2001). «The validity of evidence-based practice in social work: A reply to Stephen Webb», British Journal of Social Work, vol. 31, 801-809.

SHIN, J. et G. E. McCLOMB (1998). «Top executive leadership and organizational innovation: An empirical investigation of nonprofit human service organizations (HSOs)", Administration in Social Work, vol. 22, no 3, 1-21.

STATISTIQUE CANADA (1996). Growing up in Canada: National Longitudinal Survey of Children and Youth, Ottawa, Développement des ressources humaines du Canada.

SWIFT, K. (1995). «Manufacturing 'Bad Mothers': A critical perspective on child neglect», Toronto, University of Toronto Press.

The ARA Consulting Group Inc. (1998). Child welfare accountability review: Final report, document présenté au ministère des Services sociaux et communautaires.

TITTERTON, M. (1999). «Training professionals in risk assessment and risk management: What does the research tell us?», dans P. Parrsloe , éd., Risk Assessment in Social Care and Social Work, London, Jessica Kingsley Publishers.

TROCMÉ, N. et al. (2000). Étude canadienne sur l'incidence des signalements de cas de violence et de négligence envers les enfants. Rapport final, Santé Canada.

TROCMÉ, N., B. MERTINS-KIRKWOOD, R. MacFADDEN, R.ALAGGIA et D. GOODMAN (1999). Ontario risk assessment model phase 1: Implementation and training, Centre for Applied Social Research, Bell Canada Child Welfare Research Unit - Ministry of Community and Social Services.

USHER, C. L., D. A. GIBBS, et J. B.WILDFIRE (1995). «A framework for planning, implementing, and evaluating child welfare reforms», Child Welfare, vol. LXXIV, no 4, 859-876.

WEBB, S. A. (2001). «Some considerations on the validity of evidence-based practice in social work», British Journal of Social Work, vol. 31, 57-79. 
WILKES, J, R. (2002). «Introduction», dans Sparrow Lake Alliance, Permanency Planning in the Child Welfare, Children in Limbo Task Force.

WIMPFHEIMER, S. (1993). «Introduction to the series on liability», Administration in Social Work, vol. 17 , no 4, 1-9.

\section{Notes}

1. Voir Aronson et Sammon 2000; Cloutier et al. 2001; Paquet et al. 2001.

2. Au sujet de la couverture médiatique, voir Carter 2000; Cicchinelli 1995; Davies et al. 1999; Gold et al. 2001. Sur les transformations sociétales et les surcharges bureaucratiques, voir Cohen 1999; Aronson et Sammon 2000.

3. Vu la surreprésentation des femmes en service social, et particulièrement dans le champ de pratique de la protection des enfants, les auteures ont opté pour féminiser cet article.

4. Voir Callahan 2001; Davies et al. 1999; Swift 1995.

5. Sur le manque de justification, voir Johnson et al. 2002; Osmo et Rosen 2002. Sur la subjectivité et l'inconsistance, voir Cicchinelli 1995; DePanfilis 1996; Macmillan et al. 2002.

6. «Les décisions sont (ici) définies comme des choix intentionnels faits à partir de multiples alternatives possibles qui vont conduire à des résultats» (Van Bemmel et Helder, cité dans Proctor 2002:3).

7. Voir Martinez-Brawley et Zorita 1998.

8. Voir Camilleri 1999; Cash 2001; Martinez-Brawley et Zorita 1998; Sargent 1999.

9. Pupille de la SAE: Que l'enfant soit confié, en qualité de pupille, aux soins et à la garde de la société, pendant une période précise ne dépassant pas 12 mois (LSEF, 2000).

10. Pupille de la Couronne: Que l'enfant soit confié à la Couronne, en qualité de pupille, jusqu'à ce que la tutelle soit terminée aux termes de l'article 65, ou jusqu'à ce qu'elle prenne fin en vertu du paragraphe 71(1), et que l'enfant soit confié aux soins de la société (LSEF, 2000).

11. Voir LBEC 2002; Norgaard et Balla 2002.

12. Voir Flynn et al. 2001; LBEC 2002; Jackson 1998.

13. Voir Flynn et al. 2001; Garrett 1999;Jackson 1998; Knight et Caveney 1998; LBEC 2002; Norgaard et Balla 2002.

14. Le nombre d'enquêtes est de $20 \%$ selon Hurwitz et Cresswell (2001).

15. Le nombre d'enquêtes corroborées est de $27 \%$ selon OACAS (2001).

16. L'accroissement des enfants pris en charge est de $36 \%$ selon OACAS (2001).

17. La surcharge de tâches bureaucratiques est de $30 \%$ de leur temps au contact direct avec les clients selon Hurwitz et Cresswell (2001).

18. Voir Davidson 1990; Shin et McClomb 1998; Usher et al. 1995.

19. Voir Cohen 1999; Cohen et Austin 1994; Gummer 1995; Pine et al. 1998.

20. Voir Carter 2000; Cohen 1999; Schaffner-Goldberg 1995.

21. Voir Baird et al. 1999; Corby 1996; DePanfilis et Zuravin 1999; Lyons et al. 1996; Macmillan et al. 2002.

22. Voir DePanfilis 1996 ; Macmillan et al. 2002.

23. Voir Beaumont 1999; Macmillan et al. 2002; Cicchinelli 1995. 
24. Voir Beaumont 1999; Callahan 2001; Cicchinelli 1995; Gold, 1999; Lyons et al., 1996; Macmillan et al. 2002 ; Sargent 1999; Trocmé et al. 1999.

25. Voir Callahan, 2001; Corby 1996; Macmillan et al. 2002.

26. Voir Corby 1996; Macmillan et al. 2002; ORAM 2000; Titterton 1999.

27. Voir Knight et Caveney 1998; Jackson 1998.

28. Voir Knight et Caveney 1998; Garrett 1999.

29. Voir Knight et Caveney 1998; Garrett 1999.

30. Voir Burke 1999; Gambrill et Shlonsky 2001.

31. Voir Camilleri 1999; Cash 2001; Martinez-Brawley et Zorita 1998; Sargent 1999; Webb 2001. 